\title{
Evolving Treatment Paradigm in the Treatment of Locally Advanced Rectal Cancer
}

\author{
Clayton A. Smith, MD, PhD, and Lisa A. Kachnic, MD
}

\begin{abstract}
Locally advanced rectal cancer (LARC) carries higher risks of local and distant recurrence when treated with surgical resection alone. Multiple treatment strategies have been investigated to reduce recurrence risk and improve survival. Currently, there are 3 primary strategies for managing LARC: (1) preoperative long-course radiotherapy (RT) combined with radiosensitizing chemotherapy, which is better tolerated than postoperative chemoradiotherapy and provides tumor downstaging and improved pathologic complete response (pCR), followed by postoperative chemotherapy; (2) preoperative short-course RT alone as an alternative strategy for reducing the risk of local recurrence, followed by adjuvant postoperative chemotherapy; and (3) total neoadjuvant therapy with induction chemotherapy followed by chemoradiotherapy to improve $\mathrm{pCR}$ and reduce the difficulty of delivering chemotherapy in the postoperative setting. In addition to these currently recommended treatment paradigms, promising new strategies are available for treatment reduction. Neoadjuvant chemotherapy alone may allow for omission of RT in select patients with favorable LARC. For patients who have complete clinical responses to neoadjuvant chemotherapy and RT, nonoperative management is being considered for sphincter preservation, with surgery used as salvage. These are active areas of investigation in both institutional and cooperative group trials. The results are anticipated to provide better tailoring of neoadjuvant therapy based on patient tumor and disease response characteristics.
\end{abstract}

The incidence of rectal cancer in the United States was estimated to be 39,910 in 2017 , with approximately 50,260 deaths from combined colon and rectal cancer. ${ }^{1}$ Although surgical resection remains the definitive treatment for rectal cancer, historical rates of local failure following surgery alone using older techniques were $\geq 25 \%$. $^{2}$ The development of total mesorectal excision (TME) with sharp dissection of mesorectal contents further reduced the rates of local recurrence to as low as $8 \% .^{3}$ Despite this improvement, patients with locally advanced rectal cancer (LARC; commonly defined as T3 or T4 primary or nodal metastases) still had unacceptably high risks of local and distant failure relative to those with early-stage disease. Therefore, additional treatment strategies were examined, including the use of adjuvant radiotherapy (RT) and chemotherapy with

aDivision of Radiation Oncology, University of South Alabama Mitchell Cancer Institute, Mobile, Alabama, and 'Department of Radiation Oncology, Vanderbilt University Medical Center, Nashville, Tennessee. Submitted January 10, 2018; accepted for publication April 10, 2018. The authors have disclosed that they have no financial interests, arrangements, affiliations, or commercial interests with the the intent to reduce the rate of local and distant failure, respectively. Adjuvant RT alone decreased the risk of local recurrence without significant benefit to overall survival (OS). ${ }^{4}$ Adjuvant chemotherapy both reduced the rate of distant metastases and improved disease-free survival (DFS). ${ }^{5,6}$ This review focuses on the current treatment paradigms for LARC and highlights clinical trials that may lead to a more selective evolution in management.

\section{Neoadjuvant Versus Adjuvant RT}

Historically, the standard paradigm for the management of LARC (cT3, cT4, or node-positive disease) was TME with either low anterior resection or abdominoperineal resection (APR) for proximal and distal cancers, respec-

manufacturers of any products discussed in this article or their competitors.

Correspondence: Lisa A. Kachnic, MD, Department of Radiation Oncology, Vanderbilt University Medical Center, 2220 Pierce Avenue, Preston Research Building B-1003, Nashville, TN 37232.

Email: Lisa.a.kachnic@vanderbilt.edu 
tively. This was typically followed by adjuvant RT with or without chemotherapy and additional adjuvant multiagent chemotherapy. Due to patient difficulty in completing postoperative treatment, several trials investigated whether neoadjuvant therapy could offer similar disease control while resulting in less morbidity. In the German Rectal Cancer Study CAO/ARO/AIO-94, more than 800 patients with LARC were randomly assigned to either neoadjuvant RT to 5,040 cGy in 28 fractions with continuous-infusion 5-fluorouracil (5-FU) or to postoperative RT to 5,580 cGy in 31 fractions with $5-\mathrm{FU}^{7}$; this is commonly referred to as long-course, or standard, chemoradiotherapy (chemoRT). Surgical resection was TME, and both groups received 4 cycles of adjuvant 5 -FU. With long-term follow-up, neoadjuvant RT decreased local failure (7.1\% vs $10.1 \% ; P=.048)$ without impacting OS $(59.6 \%$ vs $59.9 \% ; P=.85)$ or distant failure $(29.8 \%$ vs $29.6 \% ; P=.9) .{ }^{8}$ Acute grade $\geq 3$ toxicity ( $27 \%$ vs $40 \% ; P=.001)$ and late grade $\geq 3$ toxicity $(14 \%$ vs $24 \% ; P=.01)$ were reduced with neoadjuvant chemoRT. Upon review of resected tumors in the neoadjuvant therapy group, 9\% were found to have pathologic complete response (pCR), which has been associated with improved survival and outcomes. ${ }^{9,10}$
The EORTC 22921 and the Fédération Francophone de Cancérologie Digestive (FFCD) 9203 trials compared outcomes for neoadjuvant chemoRT versus RT alone ( \pm adjuvant chemotherapy) in patients with clinical T3 or T4 disease. ${ }^{11-13}$ The addition of chemotherapy to neoadjuvant RT improved rates of 3 -year local control $(92.3 \%$ vs $84.7 \% ; P<.0001)$ but not 5 -year OS $(66.3 \%$ vs $65.9 \% ; P=.66)$ or 3 -year distant progression-free survival $(71.3 \%$ vs $70.7 \%$; $P=.5)$ in a pooled analysis of the data. ${ }^{14} \mathrm{pCR}$ rates were significantly improved with chemoRT $(11.2 \%$ vs $3.7 \% ; P<.0001)$, consistent with the German trial results. Collectively, these data (Table 1) support a main recommendation by NCCN Clinical Practice Guidelines in Oncology (NCCN Guidelines) for Rectal Cancer for locally advanced disease: neoadjuvant chemoRT, followed by definitive TME 5 to 12 weeks later, and then further adjuvant chemotherapy. ${ }^{15}$

\section{Short-Course RT}

Although combined chemotherapy and long-course RT has been the primary neoadjuvant treatment approach in the United States, an alternative approach in other countries and some US centers has

\begin{tabular}{|c|c|c|c|c|c|}
\hline Trial & Treatment Regimens & $\begin{array}{l}\text { Local Failure } \\
(\%)\end{array}$ & $\begin{array}{l}\text { Disease-Free } \\
\text { Survival } \\
(\%)\end{array}$ & $\begin{array}{c}\text { Overall } \\
\text { Survival } \\
\text { (\%) }\end{array}$ & $\begin{array}{l}\text { Pathologic Complete } \\
\text { Response } \\
\text { (\%) }\end{array}$ \\
\hline \multirow[t]{2}{*}{ German CAO/ARO/AIO-947,8 } & LC RT/5-FU & $7.1^{\mathrm{a}}$ & 68 & 60 & 9 \\
\hline & Adjuvant LC RT/5-FU & 10.1 & 68 & 60 & NA \\
\hline \multirow{2}{*}{ EORTC 22921/FFCD 9203 } & LC RT & 15 & 61 & 66 & 3.7 \\
\hline & LC RT/5-FU & $8^{\mathrm{a}}$ & 64 & 66 & $11.2^{\mathrm{a}}$ \\
\hline \multirow[t]{2}{*}{ Dutch TME ${ }^{17,18}$} & SC RT & $5^{\mathrm{a}}$ & NR & 48 & NR \\
\hline & TME alone & 11 & NR & 49 & NR \\
\hline \multirow[t]{2}{*}{ Bujko et al ${ }^{19}$} & LC RT/5-FU & 14.2 & 55.6 & 66.2 & $16.1^{\mathrm{a}}$ \\
\hline & SC RT & 9 & 58.4 & 67.2 & 0.7 \\
\hline \multirow[t]{2}{*}{ TROG $01.04^{20}$} & SC RT & 8 & NR & 74 & 1 \\
\hline & LC RT/5-FU & 4 & NR & 70 & $15^{\mathrm{a}}$ \\
\hline \multirow[t]{3}{*}{ Stockholm IIII2,23 } & SC RT 1-wk delay & \multirow{3}{*}{ (All arms $<5 \%$ ) } & 65 & 73 & 1.7 \\
\hline & SC RT 4- to 8-wk delay & & 64 & 76 & $10.1^{\mathrm{a}}$ \\
\hline & LC RT 4- to 8-wk delay & & 65 & 78 & NR \\
\hline \multirow[t]{2}{*}{ Spanish GCR-3 $3^{40}$} & LC RT/CAPEOX & 2 & 64 & 78 & 13 \\
\hline & $\begin{array}{l}\text { CAPEOX } \rightarrow \text { LC RT/ } \\
\text { CAPEOX }\end{array}$ & 5 & 62 & 75 & 14 \\
\hline
\end{tabular}

Abbreviations: 5-FU, 5-fluorouracil; LC, long-course; NA, not applicable; NR, not reported; RT, radiotherapy; SC, short-course; TME, total mesorectal excision.

aSignificant difference between arms. 
been neoadjuvant short-course RT given as 5 daily fractions of 5 Gy. The Swedish Rectal Cancer Trial was conducted in the pre-TME era and compared preoperative short-course RT followed by resection 1 week later versus surgery alone. With long-term follow-up, short-course RT demonstrated improved OS (38\% vs $30 \% ; P=.008)$ with reduced local failure (9\% vs $26 \% ; \mathrm{P}<.001) .{ }^{16}$ As noted, because TME was not a requirement for this trial, it was questioned whether outcomes for the surgical control arm were worse due to inferior surgical techniques. This theory was tested in the Dutch TME trial that similarly compared neoadjuvant short-course RT versus surgery alone, with TME required for all patients. ${ }^{17}$ As with trials of neoadjuvant long-course RT followed by TME, short-course RT demonstrated an improved 10 -year local failure rate ( $5 \%$ vs $11 \% ; P<.0001$ ) but no improvement in 10 -year OS rate (48\% vs $49 \%$; $P=.86) .{ }^{18}$ Because the patient population contained almost two-thirds stage I/II disease, it is likely that many patients would not have received benefit from RT. This was supported by a subset analysis of patients with stage III disease and negative circumferential resection margins (CRM) showing an OS benefit with short-course RT ( $50 \%$ vs $40 \% ; P=.032$ ).

Because of variation in practice patterns with the use of neoadjuvant long-course chemoRT or short-course RT alone for LARC, additional trials have been conducted to compare these regimens. ${ }^{19,20}$ Bujko et $\mathrm{al}^{21}$ randomized $>300$ patients to longcourse chemoRT with 5 -FU followed by resection 4 to 6 weeks later or to short-course RT followed by resection within 1 week. No differences were seen in rates of 4 -year local failure (14.2\% vs $9 \% ; P=.17)$, OS (66.2\% vs $67.2 \% ; P=.96)$, DFS ( $55.6 \%$ vs $58.4 \%$; $P=.82)$, or late toxicity $(7.1 \%$ vs $10.1 \% ; P=.36)$; $\mathrm{pCR}$ rates were significantly lower in the short-course arm $(0.7 \%$ vs $16.1 \% ; P<.001) .{ }^{19}$ Despite these pathologic findings, no differences were seen in the rate of sphincter-preservation surgery among patients treated with short-course versus standard chemoRT (61.2\% vs $58 \%$; $P=.57)$. The TROG 01.04 trial similarly randomized patients with cT3 disease to neoadjuvant short-course RT or long-course chemoRT with 5-FU followed by resection and adjuvant chemotherapy ${ }^{20}$; it also found no differences in clinical outcomes (local failure, distant failure, OS, or late toxicity) between arms, and the pCR rate was significantly less after short-course RT and short-interval surgery $(1 \%$ vs $15 \% ; P=$ not reported $)$. The results of these randomized trials of short-course RT versus long-course chemoRT found no difference in rates of APR between arms, although the short-course arm in the TROG trial had a greater number of patients with distal $(<5 \mathrm{~cm})$ cancers.

These inferior pathologic results could be attributed to differences in RT fractionation schedules or to differences in the delay between neoadjuvant therapy and surgery. The Stockholm III trial assessed the effect of delay by randomizing patients to short-course RT and surgery 1 week later versus short-course RT with a 4- to 8-week delay, as well as a third arm of long-course RT alone with a 4- to 8 -week delay. In comparing the short-course outcomes, $\mathrm{pCR}$ rates were higher with the longer delay (10.1\% vs $1.7 \% ; P<.001) .{ }^{22}$ No differences were seen in local/distant failure or OS between arms; however, patients receiving short-course RT with a 1-week delay had significantly greater incidence of postoperative complications compared with those receiving short-course RT with a 4- to 8-week delay (53\% vs $41 \% ; P=.001){ }^{23}$

The NCCN Guidelines list short-course RT as an acceptable neoadjuvant treatment option for patients with LARC and clinical T3 CRM uninvolved or node-positive disease. ${ }^{15}$ In patients with clinical T4 disease, involved CRM, or locally unresectable/ medically inoperable tumors, long-course chemoRT with or without more intensive neoadjuvant chemotherapy is recommended given the available data for pathologic downstaging with standard chemoRT.

\section{Selection of Concurrent and Adjuvant Chemotherapy}

The appropriate choice of concurrent chemotherapy was tested in the phase III randomized National Surgical Adjuvant Breast and Bowel Project trial R-04. Patients with stage II or III rectal cancer were randomized in a $2 \times 2$ design to either continuous infusion 5-FU or capecitabine with or without oxaliplatin given concurrently with long-course RT. ${ }^{24,25} \mathrm{No}$ differences were seen in rates of 5-year OS $(79.9 \%$ vs $80.8 \% ; P=.61)$, DFS ( $66.4 \%$ vs $67.7 \% ; P=.70)$, or 3 -year locoregional failure $(11.2 \%$ vs $11.8 \% ; P=.98)$ between patients treated with $5-\mathrm{FU}$ or capecitabine. Similarly, the addition of oxaliplatin did not improve OS, DFS, or locoregional failure; however, 
oxaliplatin significantly increased grade $\geq 3$ acute diarrhea $(P<.0001)$. The NCCN Guidelines recommend single-agent fluoropyrimidine-based chemotherapy as the preferred concurrent systemic agent with long-course RT. ${ }^{15}$

For patients with high-risk stage II or III colon cancer, large randomized trials have demonstrated a DFS benefit with the addition of oxaliplatin to 5-FUbased adjuvant chemotherapy. ${ }^{26-28}$ Randomized trials of patients with LARC treated with neoadjuvant chemoRT have provided mixed results regarding whether adjuvant 5-FU-based or multiagent chemotherapy improves survival. In the EORTC 22921 trial, an additional comparison of the $2 \times 2$ factorial design was randomization between 4 cycles of adjuvant 5 -FU or observation. Adjuvant 5-FU was not found to improve 10 -year OS compared with observation (51.8\% vs $48.4 \%$; $P=.32) .{ }^{13}$ Although early analysis demonstrated a DFS benefit of adjuvant therapy for patients with ypT0-2 (hazard ratio [HR], 0.64; 95\% CI, 0.45$0.91 ; P=.13)$, this benefit was not observed with longterm follow-up (HR, 0.89; 95\% CI, 0.66-1.21; $P=$ not reported). ${ }^{13,29}$ In a similar single randomization study, the Italian I-CNR-RT trial treated 655 patients with chemoRT with 5-FU, surgery, and either observation or 6 cycles of adjuvant 5-FU. For patients who underwent resection, no difference was seen in 5-year OS (70\% vs $69 \% ; P=.772$ ) or distant failure (20\% both arms).$^{30} \mathrm{~A}$ criticism of these trials is that randomization occurred before neoadjuvant therapy, which may have resulted in imbalances in pathologic staging between groups prior to receiving adjuvant therapy. ${ }^{31}$ Additionally, only $43 \%$ to $60 \%$ of patients received adjuvant chemotherapy as prescribed.

Several randomized trials have investigated the effect of adding oxaliplatin to 5-FU-based therapy. The German CAO/ARO/AIO-04 trial, which randomized $>1,200$ patients to either standard chemoRT with 5-FU followed by adjuvant 5-FU, or to the same treatment with the addition of oxaliplatin to both neoadjuvant and adjuvant therapy, demonstrated that 3-year DFS was significantly improved with the addition of oxaliplatin ( $76 \%$ vs $71 \% ; P=.03) .{ }^{32}$ The ADORE phase II trial had a similar design, with randomization of patients with pathologic stage II or III to either adjuvant 5-FU or FOLFOX chemotherapy, and similarly showed that the 3 -year DFS rate was increased in patients receiving oxaliplatin ( $72 \%$ vs $63 \%$; $P=.047) .{ }^{33} \mathrm{On}$ subset analysis, this benefit appeared to be for patients with pathologic stage III disease. In contrast to these results, the PETACC- 6 trial of patients randomized to chemoRT with capecitabine and adjuvant capecitabine or to the same treatment with the addition of neoadjuvant and adjuvant oxaliplatin did not find a difference in 3-year DFS $(76.5 \%$ vs $75.4 \% ; P=.744) .{ }^{34}$

Several meta-analyses using either aggregate or individual patient data have not found an improvement in OS or DFS when testing the effect of adjuvant chemotherapy versus observation, or the effect of adding oxaliplatin to 5-FU-based therapy. ${ }^{35-37} \mathrm{De}$ spite a lack of conclusive evidence of benefit, sufficient data show a benefit for some patients with LARC that the NCCN panel continues to recommend adjuvant FOLFOX, CAPEOX, or single-agent fluoropyrimidine-based chemotherapy ${ }^{15}$ The recommended total duration of perioperative therapy for patients with LARC is 6 months.

\section{Induction Chemotherapy and ChemoRT}

Most patients with LARC will receive neoadjuvant chemoRT or short-course RT followed by surgical resection and adjuvant chemotherapy as the standard of care. There are reports that pathologic downstaging after neoadjuvant therapy is associated with improved survival outcomes. One measure of pathologic downstaging has been $\mathrm{pCR}$ rates, although more recently the neoadjuvant rectal (NAR) score has been used as a surrogate for clinical trial outcomes. ${ }^{9,10,38,39}$ Several trials have now addressed sequencing of adjuvant therapy with the goals of improving $\mathrm{pCR}$ rates/NAR scores, ensuring completion of prescribed therapy, and reducing late toxicity.

The Spanish GCR-3 trial investigated moving adjuvant chemotherapy up-front as induction prior to chemoRT. Patients were randomized to longcourse chemoRT with CAPEOX followed by TME and adjuvant CAPEOX or to induction CAPEOX followed by chemoRT and TME. ${ }^{40}$ No differences were seen in rates of 5 -year local failure ( $2 \%$ vs $5 \%$; $P=.61)$, distant failure ( $21 \%$ vs $23 \% ; P=.79)$, OS ( $78 \%$ vs $75 \% ; P=.64)$, or $\mathrm{pCR}(13 \%$ vs $14 \% ; P=.94)$. This demonstrated the safety of starting with induction chemotherapy prior to standard neoadjuvant chemoRT and surgery.

Garcia-Aguilar et $\mathrm{al}^{41}$ performed a multi-institutional nonrandomized phase II trial testing the effect 
of increasing preoperative chemotherapy cycles on pCR. Patients received chemoRT with 5-FU followed by (1) TME, (2) 2 cycles of mFOLFOX6 then TME, (3) 4 cycles of mFOLFOX6 then TME, or (4) 6 cycles of mFOLFOX 6 and TME. Increasing the number of cycles of neoadjuvant mFOLFOX6 produced higher pCR rates (groups $1-4: 18 \%$ vs $25 \%$ vs $30 \%$ vs $38 \%$, respectively; $P=.0036$ ). One consequence of lengthening the interval between chemoRT and surgery was an increase in pelvic fibrosis at the time of surgery; however, this did not impact the $\mathrm{R} 0$ resection rate. Publication of clinical outcomes is pending.

These trials highlight the safety and potentially improved efficacy of a total neoadjuvant therapy approach. NRG-GI002 (ClinicalTrials.gov identifier: NCT02921256) is a randomized phase II trial platform investigating novel neoadjuvant strategies to produce a reduction in NAR scores as surrogate for OS in patients at particularly high risk of locoregional recurrence. The intent is that successful arms may go on to phase III testing. The standard arm is mFOLFOX6 x 8 cycles followed by capecitabine/ RT and surgery. The first experimental arm added the PARP inhibitor veliparib to capecitabine/RT and is now closed to accrual. Study eligibility criteria select for patients with higher-risk disease than those on the Alliance PROSPECT trial (ClinicalTrials.gov identifier: NCT01515787), including: (1) cT3-4 and distal location $(<5 \mathrm{~cm}$ from anal verge); (2) bulky cT4 tumor within $3 \mathrm{~mm}$ of the mesorectal fascia; (3) cN2 disease; or (4) not a candidate for sphincter preservation prior to preoperative therapy. This may allow identification of new neoadjuvant therapies for patients with more aggressive LARC.

\section{Preoperative Chemotherapy Alone}

Because the benefit of neoadjuvant RT is primarily in reducing local failure, some investigators have questioned whether RT may be safely omitted and replaced by neoadjuvant chemotherapy alone. The Chinese FOWARC trial randomized 495 patients to 1 of 3 arms: (1) long-course chemoRT with 5-FU and postoperative 5 -FU, (2) chemoRT with mFOLFOX6 and postoperative mFOLFOX6, or (3) neoadjuvant mFOLFOX6 alone (no RT) and postoperative mFOLFOX6. The patient population included cT3-4 or $\mathrm{CN} 1-2$ disease, and approximately $50 \%$ of patients had distal tumors $<5 \mathrm{~cm}$ from the anal verge. The likelihood of RO resection or sphincter-preservation surgery was similar regardless of neoadjuvant regimen; however, there was a significant difference in $\mathrm{pCR}$ rate depending on treatment arm, with arm 3 (neoadjuvant mFOLFOX6 alone) having the lowest rate (arms $1-3$ : $14 \%$ vs $27.5 \%$ vs $6.6 \%$, respectively; $P=.005$, for arm 1 vs arm 2). ${ }^{42}$ In comparing the RT arms, mFOLFOX6 combined with RT produced greater grade $3 / 4$ gastrointestinal toxicity over standard RT +5 -FU ( $14.5 \%$ vs $7.7 \%$ diarrhea; $P=$ not reported).

The Alliance PROSPECT trial was opened in 2012 to assess whether patients with favorable LARC may be treated with neoadjuvant chemotherapy alone (ClinicalTrials.gov identifier: NCT01515787). The study population includes patients with cT2N1 or cT3NO-1 disease and sphincter-preservation surgical candidates, while excluding cT4, cN2, or distal tumors that would require APR. Patients are randomized to chemoRT with 5-FU followed by resection or to induction FOLFOX6 followed by response assessment. Patients in the experimental chemotherapy-alone arm with $>20 \%$ imaging response undergo resection, whereas nonresponders receive chemoRT before surgery. Both arms receive adjuvant chemotherapy. Compared with the FOWARC study, the PROSPECT patient population is selected for tumors with more favorable characteristics that may have a lower risk of local recurrence or need for downstaging prior to surgery. Clinical outcomes from both trials are awaited before neoadjuvant chemotherapy alone without RT can be considered an option for select patients with LARC; this treatment paradigm is investigational and not currently recommended by NCCN. ${ }^{15}$

\section{Nonoperative Management}

Changes in neoadjuvant management with improved pCR rates have prompted investigation into whether surgery may be safely omitted in select patients. This would carry the potential advantage of sphincter preservation and avoidance of surgical morbidity. This approach of omitting surgery has been referred to as "nonoperative management," "watchful waiting," or "watch-and-wait." One of the earliest reports of this approach by Habr-Gama et $\mathrm{al}^{43}$ described a retrospective series of 265 patients with distal nonmetastatic rectal cancers treated with long-course chemoRT. Patients with no evidence of disease on restaging by digi- 
tal examination, endoscopy with biopsy, and CT imaging underwent nonoperative management with close surveillance; patients with evidence of residual disease underwent definitive TME. Of the cohort, 27\% had clinical complete response (cCR), whereas $8 \%$ who underwent surgery had pCR. The cCR group had an increased 5 -year OS rate ( $100 \%$ vs $88 \% ; P=.01)$, but no difference was seen in rates of DFS ( $92 \%$ vs $83 \%$; $P=.09$ ) or local failure ( $7 \%$ vs $13.6 \% ; P=.2$ ). Because $27 \%$ of patients in this study had a cCR, which is higher than the approximately $15 \%$ pCR rates reported by many trials, this suggests that increasing time between completion of chemoRT and surgery has a significant effect on pathologic tumor response. This is supported by the results of the Stockholm III trial. ${ }^{22}$ These results also suggest that nonoperative management may allow for organ preservation without increased risk of recurrence or death in carefully selected patients.

Martens et $\mathrm{al}^{44}$ reported outcomes from a prospective cohort of patients managed with either nonoperative management or transanal excision after $\mathrm{cCR}$ or near $\mathrm{CCR}$ as defined by digital examination, endoscopy, and MRI. Of 100 patients, 61 had an initial cCR 8 weeks after neoadjuvant therapy, 24 were found to have $\mathrm{cCR}$ at a second assessment 3 months later, and the remaining 15 patients underwent transanal excision. Overall, $15 \%$ of patients developed locoregional failures and were salvaged with surgery; 3 -year OS was $97 \%$, distant metastasis-free survival was $97 \%$, and colostomy-free survival was $95 \%$. In addition to these findings, numerous retrospective and prospective cohort studies have reported favorable outcomes in patients undergoing nonoperative management with chemoRT. ${ }^{44-49}$ Reported local failure rates range from $15 \%$ to $38 \%$, but importantly, salvage therapies were successful for $>90 \%$ of patients with local failure.
Reported 5-year OS rates with this nonoperative approach range from $96 \%$ to $100 \%$.

Nonoperative management continues to be an active area of investigation. Many of the trials reported involve very careful patient selection that can bias results, which prompts caution against it as a standard treatment approach for most patients. Because patient selection for nonoperative management after chemoRT can be difficult and depends on accurate clinical and diagnostic assessment, its use has primarily been limited to high-volume centers with experienced multidisciplinary teams. This is likely to evolve as diagnostic modalities are better able to predict for pathologic response. At this time, the NCCN Guidelines do not recommend nonoperative management in patients with LARC who are otherwise considered candidates for surgical resection. ${ }^{15}$

\section{Conclusions}

The treatment paradigm for patients with LARC has evolved over the past decades from resection alone to postoperative adjuvant therapy, and now to neoadjuvant chemoRT followed by TME and additional chemotherapy, resulting in dramatically decreased local failure rates. Continued refinement of treatment strategies with total neoadjuvant chemotherapy and chemoRT in patients at higher risk of recurrence has also improved pCR rates. Active clinical trials are aimed at further tailoring treatment to disease risk, including selecting patients who may be at a lower risk of recurrence to undergo neoadjuvant chemotherapy alone, those at increased risk of recurrence to undergo additional intensified and targeted neoadjuvant regimens, and those with a cCR to neoadjuvant chemoRT to undergo nonoperative management.

\section{References}

1. Key Statistics for Colorectal Cancer. American Cancer Society Website. Available at: https://www.cancer.org/cancer/colon-rectal-cancer/about/keystatistics.html. Accessed November 28, 2017.

2. McCall JL, Cox MR, Wattchow DA. Analysis of local recurrence rates after surgery alone for rectal cancer. Int J Colorectal Dis 1995;10:126-132.

3. Heald RJ, Moran BJ, Ryall RD, et al. Rectal cancer: the Basingstoke experience of total mesorectal excision, 1978-1997. Arch Surg 1998;133:894-899.

4. Colorectal Cancer Collaborative Group. Adjuvant radiotherapy for rectal cancer: a systematic overview of 8,507 patients from 22 randomised trials. Lancet 2001;358:1291-1304.

5. Thomas PR, Lindblad AS. Adjuvant postoperative radiotherapy and chemotherapy in rectal carcinoma: a review of the Gastrointestinal Tumor Study Group experience. Radiother Oncol 1988;13:245-252.

6. Krook JE, Moertel CG, Gunderson LL, et al. Effective surgical adjuvan therapy for high-risk rectal carcinoma. N Engl J Med 1991;324:709-715.

7. Sauer R, Becker H, Hohenberger W, et al. Preoperative versus postoperative chemoradiotherapy for rectal cancer. N Engl J Med 2004;351:1731-1740.

8. Sauer R, Liersch T, Merkel S, et al. Preoperative versus postoperative chemoradiotherapy for locally advanced rectal cancer: results of the German CAO/ARO/AIO-94 randomized phase III trial after a median follow-up of 11 years. J Clin Oncol 2012;30:1926-1933.

9. Martin ST, Heneghan HM, Winter DC. Systematic review and metaanalysis of outcomes following pathological complete response to neoadjuvant chemoradiotherapy for rectal cancer. Br J Surg 2012;99:918928.

10. Zorcolo L, Rosman AS, Restivo A, et al. Complete pathologic response after combined modality treatment for rectal cancer and long-term survival: a meta-analysis. Ann Surg Oncol 2012;19:2822-2832. 
Evolving Treatment of Advanced Rectal Cancer

11. Gérard JP, Conroy T, Bonnetain F, et al. Preoperative radiotherapy with or without concurrent fluorouracil and leucovorin in T3-4 rectal cancers: results of FFCD 9203. J Clin Oncol 2006;24:4620-4625.

12. Bosset JF, Collette L, Calais G, et al. Chemotherapy with preoperative radiotherapy in rectal cancer. N Engl J Med 2006;355:1114-1123.

13. Bosset JF, Calais G, Mineur L, et al. Fluorouracil-based adjuvant chemotherapy after preoperative chemoradiotherapy in rectal cancer: long-term results of the EORTC 22921 randomised study. Lancet Oncol 2014;15:184-190.

14. Bonnetain F, Bosset JF, Gerard JP, et al. What is the clinical benefit of preoperative chemoradiotherapy with 5FU/leucovorin for T3-4 rectal cancer in a pooled analysis of EORTC 22921 and FFCD 9203 trials: surrogacy in question? Eur J Cancer 2012;48:1781-1790.

15. Benson AB III, Venook AP, Al-Hawary MM, et al. NCCN Clinical Practice Guidelines in Oncology: Rectal Cancer. Version 1.2018. Accessed March 21, 2018. To view the most recent version of these guidelines, visit NCCN.org.

16. Folkesson J, Birgisson H, Pahlman L, et al. Swedish Rectal Cancer Trial long lasting benefits from radiotherapy on survival and local recurrence rate. J Clin Oncol 2005;23:5644-5650.

17. Kapiteijn E, Marijnen CA, Nagtegaal ID, et al. Preoperative radiotherapy combined with total mesorectal excision for resectable rectal cancer. N Engl J Med 2001;345:638-646.

18. van Gijn W, Marijnen CA, Nagtegaal ID, et al. Preoperative radiotherapy combined with total mesorectal excision for resectable rectal cancer: 12-year follow-up of the multicentre, randomised controlled TME trial. Lancet Oncol 2011;12:575-582.

19. Bujko K, Nowacki MP, Nasierowska-Guttmejer A, et al. Sphincter preservation following preoperative radiotherapy for rectal cancer: report of a randomised trial comparing short-term radiotherapy vs conventionally fractionated radiochemotherapy. Radiother Oncol 2004;72:15-24.

20. Ngan SY, Burmeister B, Fisher RJ, et al. Randomized trial of short-course radiotherapy versus long-course chemoradiation comparing rates of local recurrence in patients with T3 rectal cancer: Trans-Tasman Radiation Oncology Group trial 01.04. J Clin Oncol 2012;30:3827-3833.

21. Bujko K, Nowacki MP, Nasierowska-Guttmejer A, et al. Long-term results of a randomized trial comparing preoperative short-course radiotherapy with preoperative conventionally fractionated chemoradiation for rectal cancer. Br J Surg 2006;93:1215-1223.

22. Pettersson D, Lörinc E, Holm T, et al. Tumour regression in the randomized Stockholm III trial of radiotherapy regimens for rectal cancer. Br J Surg 2015;102:972-978.

23. Erlandsson J, Holm T, Pettersson D, et al. Optimal fractionation of preoperative radiotherapy and timing to surgery for rectal cancer (Stockholm III): a multicentre, randomised, non-blinded, phase 3, noninferiority trial. Lancet Oncol 2017;18:336-346.

24. O'Connell MJ, Colangelo LH, Beart RW, et al. Capecitabine and oxaliplatin in the preoperative multimodality treatment of rectal cancer surgical end points from National Surgical Adjuvant Breast and Bowel Project trial R-04. J Clin Oncol 2014;32:1927-1934.

25. Allegra CJ, Yothers G, O'Connell MJ, et al. Neoadjuvant 5-FU or capecitabine plus radiation with or without oxaliplatin in rectal cancer patients: a phase III randomized clinical trial. J Natl Cancer Inst 2015;107:pii: djv248.

26. Kuebler JP, Wieand HS, O'Connell MJ, et al. Oxaliplatin combined with weekly bolus fluorouracil and leucovorin as surgical adjuvant chemotherapy for stage II and III colon cancer: results from NSABP C-07. J Clin Oncol 2007;25:2198-2204.

27. André $T$, Boni $C$, Navarro $M$, et al. Improved overall survival with oxaliplatin, fluorouracil, and leucovorin as adjuvant treatment in stage II or III colon cancer in the MOSAIC trial. J Clin Oncol 2009;27:3109-3116.

28. Schmoll HJ, Tabernero J, Maroun J, et al. Capecitabine plus oxaliplatin compared with fluorouracil/folinic acid as adjuvant therapy for stage III colon cancer: final results of the NO16968 randomized controlled phase III trial. J Clin Oncol 2015;33:3733-3740.

29. Collette L, Bosset JF, den Dulk M, et al. Patients with curative resection of cT3-4 rectal cancer after preoperative radiotherapy or radiochemotherapy: does anybody benefit from adjuvant fluorouracil-based chemotherapy? A trial of the European Organisation for Research and Treatment of Cancer Radiation Oncology Group. J Clin Oncol 2007;25:4379-4386.

30. Sainato A, Cernusco Luna Nunzia V, Valentini V, et al. No benefit of adjuvant fluorouracil leucovorin chemotherapy after neoadjuvant chemoradiotherapy in locally advanced cancer of the rectum (LARC): long term results of a randomized trial (I-CNR-RT). Radiother Oncol 2014;113:223-229

31. Carvalho C, Glynne-Jones R. Challenges behind proving efficacy of adjuvant chemotherapy after preoperative chemoradiation for rectal cancer. Lancet Oncol 2017;18:e354-363.

32. Rödel C, Graeven U, Fietkau R, et al. Oxaliplatin added to fluorouracilbased preoperative chemoradiotherapy and postoperative chemotherapy of locally advanced rectal cancer (the German CAO/ARO/AIO-04 study): final results of the multicentre, open-label, randomised, phase 3 trial. Lancet Oncol 2015;16:979-989.

33. Hong YS, Nam BH, Kim K, et al. Oxaliplatin, fluorouracil, and leucovorin versus fluorouracil and leucovorin as adjuvant chemotherapy for locally advanced rectal cancer after preoperative chemoradiotherapy (ADORE): an open-label, multicentre, phase 2, randomised controlled trial. Lancet Oncol 2014;15:1245-1253.

34. Schmoll HJ, Stein A, Hofheinz RD, et al. Preoperative chemoradiotherapy and postoperative chemotherapy with capecitabine and oxaliplatin vs capecitabine alone in locally advanced rectal cancer: final analyses [abstract]. Ann Oncol 2016;27(Suppl):Abstract 467PD.

35. Breugom AJ, van Gijn W, Muller EW, et al. Adjuvant chemotherapy for rectal cancer patients treated with preoperative (chemo)radiotherapy and total mesorectal excision: a Dutch Colorectal Cancer Group (DCCG) randomized phase III trial. Ann Oncol 2015;26:696-701.

36. Bujko K, Glimelius B, Valentini V, et al. Postoperative chemotherapy in patients with rectal cancer receiving preoperative radio(chemo)therapy: a meta-analysis of randomized trials comparing surgery \pm a fluoropyrimidine and surgery + a fluoropyrimidine \pm oxaliplatin. Eur J Surg Oncol 2015;41:713-723.

37. Petrelli F, Coinu A, Lonati V, Barni S. A systematic review and metaanalysis of adjuvant chemotherapy after neoadjuvant treatment and surgery for rectal cancer. Int J Colorectal Dis 2015;30:447-457.

38. George TJ, Allegra CJ, Yothers G. Neoadjuvant rectal (NAR) score: a new surrogate endpoint in rectal cancer clinical trials. Curr Colorectal Cancer Rep 2015;11:275-280.

39. Sun Y, Zhang Y, Wu X, et al. Prognostic significance of neoadjuvant rectal score in locally advanced rectal cancer after neoadjuvant chemoradiotherapy and construction of a prediction model. J Surg Oncol 2018;117:737-744

40. Fernandez-Martos C, Garcia-Albeniz X, Pericay C, et al. Chemoradiation, surgery and adjuvant chemotherapy versus induction chemotherapy followed by chemoradiation and surgery: long-term results of the Spanish GCR-3 phase II randomized trial. Ann Oncol 2015;26:1722-1728.

41. Garcia-Aguilar J, Chow OS, Smith DD, et al. Effect of adding mFOLFOX6 after neoadjuvant chemoradiation in locally advanced rectal cancer: a multicentre, phase 2 trial. Lancet Oncol 2015;16:957-966.

42. Deng Y, Chi P, Lan P, et al. Modified FOLFOX6 with or without radiation versus fluorouracil and leucovorin with radiation in neoadjuvant treatment of locally advanced rectal cancer: initial results of the Chinese FOWARC multicenter, open-label, randomized three-arm phase III trial. J Clin Oncol 2016;34:3300-3307.

43. Habr-Gama A, Perez RO, Nadalin W, et al. Operative versus nonoperative treatment for stage 0 distal rectal cancer following chemoradiation therapy: long-term results. Ann Surg 2004;240:711-717, discussion 717-718.

44. Martens MH, Maas M, Heijnen LA, et al. Long-term outcome of an organ preservation program after neoadjuvant treatment for rectal cancer. J Natl Cancer Inst 2016;108:pii: djw291.

45. Appelt AL, Pløen J, Harling H, et al. High-dose chemoradiotherapy and watchful waiting for distal rectal cancer: a prospective observational study. Lancet Oncol 2015;16:919-927.

46. Smith JD, Ruby JA, Goodman KA, et al. Nonoperative management of rectal cancer with complete clinical response after neoadjuvant therapy. Ann Surg 2012;256:965-972.

47. Habr-Gama A, Gama-Rodrigues J, São Julião GP, et al. Local recurrence after complete clinical response and watch and wait in rectal cancer after neoadjuvant chemoradiation: impact of salvage therapy on local disease control. Int J Radiat Oncol Biol Phys 2014;88:822-828.

48. Renehan AG, Malcomson L, Emsley R, et al. Watch-and-wait approach versus surgical resection after chemoradiotherapy for patients with rectal cancer (the OnCoRe project): a propensity-score matched cohort analysis. Lancet Oncol 2016;17:174-183.

49. Maas M, Beets-Tan RG, Lambregts DM, et al. Wait-and-see policy for clinical complete responders after chemoradiation for rectal cancer. J Clin Oncol 2011;29:4633-4640. 